\title{
CARACTERÍSTICAS AGRONÓMICAS DEL PIMENTÓN (Capsicum annuum L.) DE TRES VARIEDADES EN EL MUNICIPIO DE VALLEDUPAR - CESAR
}

\author{
AGRONOMIC CHARACTERISTICS OF THE PEPPER (Capsicum annuUm L.) OF 3 \\ VARIETIES IN THE MUNICIPALITY OF VALLEDUPAR -CESAR
}

\section{Blanca Mercedes Gómez Guerrero}

Estudiante de agronomía Universidad Nacional Abierta y a Distancia UNAD ORCID: https://orcid.org/0000-0001-6517-7585

Correo: bmgomezgu@unadvirtual.edu.co

\section{Janer Eugenio Payares Guerrero}

Estudiante de agronomía

Universidad Nacional Abierta y a Distancia UNAD ORCID: https://orcid.org/0000-0002-8695-3739

Correo: jepayaresg@unadvirtual.edu.co

\author{
Alexander Salazar Montoya \\ Ingeniero agrónomo, especialista en gestión ambiental. \\ Universidad Nacional Abierta y a Distancia - UNAD, ECAPMA \\ ORCID: https://orcid.org/0000-0002-0504-6679 \\ Correo: alexander.salazar@unad.edu.co \\ Flor Manuela Ariza Molina \\ Contador público - UPC, especialista en finanzas - UPC, magister en \\ administración de organizaciones. - UNAD \\ Universidad Nacional Abierta y a Distancia - UNAD, ECACEN \\ ORCID: https://orcid.org/0000-0001-8046-5040 \\ Correo: flor.ariza@unad.edu.co
}

Citación: Gómez, B., Payares, J., Salazar, A., Ariza, F. (2021). Características agronómicas del pimentón (Capsicum annuum L.) de tres variedades en el Municipio de VALLEDUPAR - CESAR. Working Papers - ECAPMA, 1, 17 - 26. DOI: https://doi.org/10.22490/ECAPMA.4234 


\section{RESUMEN}

El departamento del Cesar, tiene un gran potencial para la producción de hortalizas y entre ellas están el pimentón según (ASOHOFRUCOL, 2006), que apoyaría la diversificación y generación de ingresos a pequeñas familias productoras, pero hay muy poca investigación sobre hortalizas en la región Caribe, que hace necesario impulsar este tipo de estudios que generen conocimiento y transferencia de tecnología para estos cultivos. Por lo anterior la presente investigación está orientada a determinar las características agronómicas del cultivo de ají pimentón (Capsicum annuum), de 3 variedades Cortes, Zapata y 14 pe9572 bajo las condiciones climáticas de bosque seco tropical del municipio de Valledupar - Cesar, El desarrollo de esta investigación se realizó bajo la implementación de un cultivo tecnificado con riego por goteo, buenas prácticas agrícolas que incluyen un plan de fertilización, manejo integrado de malezas y el manejo integrado de plagas y enfermedades. El estudio se realizó en unidades experimentales de 20 metros cuadrados con un diseño experimental de bloques completamente al azar con tres variedades y tres réplicas, que permitió el seguimiento y evaluación de las variables agronómicas (Número de frutos, peso en kilogramos, diámetro polar y ecuatorial) de las tres variedades de pimentón.

Los resultados obtenidos de las tres variedades, a partir del análisis estadístico con base en cada una de las variables, no reportaron diferencias significativas entre ellas, pero si se observó una buena adaptabilidad de las tres variedades bajo las condiciones agroclimáticas de Valledupar.

Palabras Clave: Diámetro polar; diámetro ecuatorial; grosor de la pulpa; fertilización; adaptación

\section{ABSTRACT}

The department of Cesar has great potential for the production of vegetables and among them are paprika according to (ASOHOFRUCOL, 2006), which would support diversification and income generation for small producer families, but there is very little research on vegetables in the region Caribbean, which makes it necessary to promote this type of study that generates knowledge and technology transfer for these crops. Therefore, this research is aimed at determining the agronomic characteristics of the cultivation of paprika pepper (Capsicum annuum), of 3 varieties Cortes, Zapata and 14pe9572 under the climatic conditions of tropical dry forest in the municipality 
of Valledupar - Cesar, The development of this The research was carried out under the implementation of a technified crop with drip irrigation, good agricultural practices that include a fertilization plan, integrated weed management and integrated pest and disease management. The study was carried out in experimental units of 20 square meters with an experimental design of completely randomized blocks with three varieties and three replicas, which will arrive at the monitoring and evaluation of the agronomic variables

\section{INTRODUCCIÓN}

El pimentón es una de las especies del género Capsicum, y hace parte de la familia de las solanáceas. Es la especie dulce más cultivada de este género. Su origen inicio en el continente americano, que comprende la parte sur de Brasil; y también se considera en Colombia como uno de los centros de origen. DANE (2015). En relación a América Latina, donde los mayores productores son México, Argentina y Venezuela; Colombia ocupa el quinto lugar con 2.484 hectáreas, 16.365 toneladas de producción y rendimientos de 11 toneladas/hectárea, registrando cada día tendencias hacia la especialización territorial produciendo cuatro variedades de ají: Tabasco, (number of fruits, weight in kilograms, polar and equatorial diameter) of the three varieties of paprika.

The results obtained from the three varieties, from the statistical analysis based on each of the variables, did not report significant differences between them, but a good adaptability of the three varieties is achieved under the agroclimatic conditions of Valledupar.

Keywords: Polar diameter; equatorial diameter; pulp thickness; fertilization; adaptation

Jalapeño, Habanero y Cayena. (Martínez, A. 2015).

Cabe resaltar que a pesar de que el cultivo de pimentón es una excelente fuente de alimento y empleo para la población, este fruto requiere unas condiciones agroclimáticas óptimas, donde la temperatura juega un papel fundamental, debido a que su desarrollo óptimo ocurre a temperaturas entre 18 y $28{ }^{\circ} \mathrm{C}$. A temperaturas más altas de $32^{\circ} \mathrm{C}$ y en condiciones de menor humedad relativa, se provocan abortos o caída de botones florales y flores, así como la capacidad de reducción del polen para la fecundación de las flores. (DANE. 2015). 
La ciudad de Valledupar está clasificada climáticamente en Bosque Seco Tropical con una temperatura que varía entre 22 a $37^{\circ} \mathrm{C}$ y rara vez baja a menos de $20^{\circ} \mathrm{C}$ o sube a más de $39^{\circ} \mathrm{C}$, (Valledupar. 2019), Esto podría ser una gran limitante para el establecimiento del cultivo de pimentón en la zona, sin embargo debido a la falta de información acerca de la adaptabilidad del cultivo de pimentón en condiciones de bosque seco tropical, se realizó este estudio que tiene como objetivo identificar las características agronómicas y capacidad de adaptación de tres variedades del pimentón (Capsicum annuum), con el fin de impulsar el desarrollo investigativo en el sector agrícola, que sería un gran aporte hacia la seguridad alimentaria, la generación de empleo y diversificación productiva. Adicionalmente se planteó esta investigación con tres variedades de pimentón (Cortes, Zapata y 14pe9572) que son la más cultivadas en el país y las de mayor posibilidad de adaptación a las condiciones agroclimáticas de Valledupar.

\section{METODOLOGÍA}

La investigación se realizó en la zona de vida de bosque seco tropical en el municipio de Valledupar - Cesar, en terrenos de la Universidad Nacional Abierta y a Distancia UNAD. 8

Esta investigación es de tipo cuantitativa basada en la utilización de técnicas estadísticas para conocer aspectos sobre una población (Hueso, A., \& Cascant, 2012). Los datos fueron recolectados a través de instrumentos de medición que se presentan en la tabla 1.

El desarrollo experimental de esta investigación se llevó a cabo por métodos y procedimientos orientados al cumplimiento del objetivo del proyecto, para identificar las características agronómicas de las tres variedades de pimentón a través de su cultivo y el seguimiento en campo desde la germinación, sostenimiento y cosecha, para medir los siguientes parámetros: 
Tabla 1. Validación de instrumentos de investigación.

\begin{tabular}{|c|c|c|c|}
\hline Objetivo & $\begin{array}{l}\text { Instrumento de } \\
\text { investigación y } \\
\text { Variables de evaluación }\end{array}$ & $\begin{array}{l}\text { Validación de } \\
\text { instrumento de } \\
\text { investigación }\end{array}$ & $\begin{array}{l}\text { Resultados } \\
\text { esperados }\end{array}$ \\
\hline $\begin{array}{l}\text { Identificar las } \\
\text { Características } \\
\text { agronómicas } \\
\text { (Diámetro polar, } \\
\text { Diámetro ecuatorial, } \\
\text { número de frutos } \\
\text { por planta, peso del fruto } \\
\text { y grosor de la pulpa) } \\
\text { /en las tres variedades } \\
\text { del pimentón } \\
\text { (Capsicum annuum } \text { L.). }\end{array}$ & $\begin{array}{l}\text { Se identifican } \\
\text { las características } \\
\text { agronómicas mediante el } \\
\text { uso de instrumentos de } \\
\text { medición como el } \\
\text { calibrador y gramera } \\
\text { digital para medir las } \\
\text { variables (Diámetro polar, } \\
\text { Diámetro ecuatorial, } \\
\text { número de frutos } \\
\text { por planta, peso del fruto } \\
\text { y grosor de la pulpa) }\end{array}$ & $\begin{array}{l}\text { Se verificó a través del } \\
\text { método de validación de } \\
\text { contenido, la cual está } \\
\text { definida según } \\
\text { Bohrnstedt, (1976) como } \\
\text { grado en el que la } \\
\text { medición representa al } \\
\text { concepto o variable } \\
\text { medida. (Sampieri, } \\
\text { s.f).Para esto se hizo una } \\
\text { revisión de cómo han sido } \\
\text { determinados estos } \\
\text { parámetros por otros } \\
\text { investigadores. }\end{array}$ & $\begin{array}{l}\text { Conocimiento de } \\
\text { las características } \\
\text { agronómicas de } \\
\text { las variedades de } \\
\text { pimentón } \\
\text { evaluadas. }\end{array}$ \\
\hline
\end{tabular}

Fuente: autores.

- Diámetro Polar: se utilizó el calibrador Vernier, para medir el fruto en centímetros desde la base del pedúnculo hasta el ápice, se tomaron los frutos recolectados en 4 plantas tomadas al azar de cada unidad experimental.

- Diámetro ecuatorial: se utilizó el calibrador Vernier en la parte media del fruto en centímetros, se tomaron los frutos recolectados en 4 plantas tomadas al azar de cada unidad experimental.

- Número de frutos por planta: se contó el número de frutos de las 4 plantas tomadas al azar de cada unidad experimental.

- Peso del fruto: se obtuvo sumando el peso total de los pimentones cosechados de cada unidad experimental y se expresó en kilogramos, se tomaron los frutos de 4 plantas al azar y se realizó la medición con la gramera.

- Grosor de la pulpa: se procedió a cortar el pimentón por la mitad con un exacto y luego con un calibrador se midió el grosor de la pulpa en milímetros, en uno de los frutos recolectados en las 4 plantas tomadas al azar de cada unidad experimental. 
Las mediciones de las variables para cada una de las variedades se realizaron cada 8 días, se iniciaron a los 11 días después de trasplante hasta los 95 días después de trasplante una vez se recolectó la cosecha. Todos los tratamientos tuvieron el mismo manejo agronómico desde la preparación del terreno, manejo de arvenses, fertilización, podas y manejo fitosanitario.

El análisis estadístico se realizó sobre las variables agronómicas del pimentón y se procesó la información obtenida en campo con la ayuda del programa Statgraphics centurión, se construyó la ANOVA para identificar los factores significativos y de allí las pruebas de rangos múltiples para conocer cuales medias son significativamente diferentes de otras.

\section{RESULTADOS Y DISCUSIÓN}

Los frutos producidos por la variedad Cortes, Zapata, y 14 pe9572 no presentaron diferencias significativas, probablemente al óptimo funcionamiento en cada uno de los procesos fisiológicos de las plantas, que cuentan con la condición de humedad necesaria para su desarrollo, que coinciden con lo publicado por Salisbury \& Ross (2000), cuando afirman que el beneficio y desarrollo de un gran número de funciones en beneficio de las plantas, depende de la disponibilidad de agua en el suelo. De tal manera que el peso de los frutos se ve directamente influenciados por la cantidad de agua suministrada a las plantas. (Montes Hernández.2017). A continuación, se presentan las figuras que resumen los resultados obtenidos en la medición de cada una de las variables. 


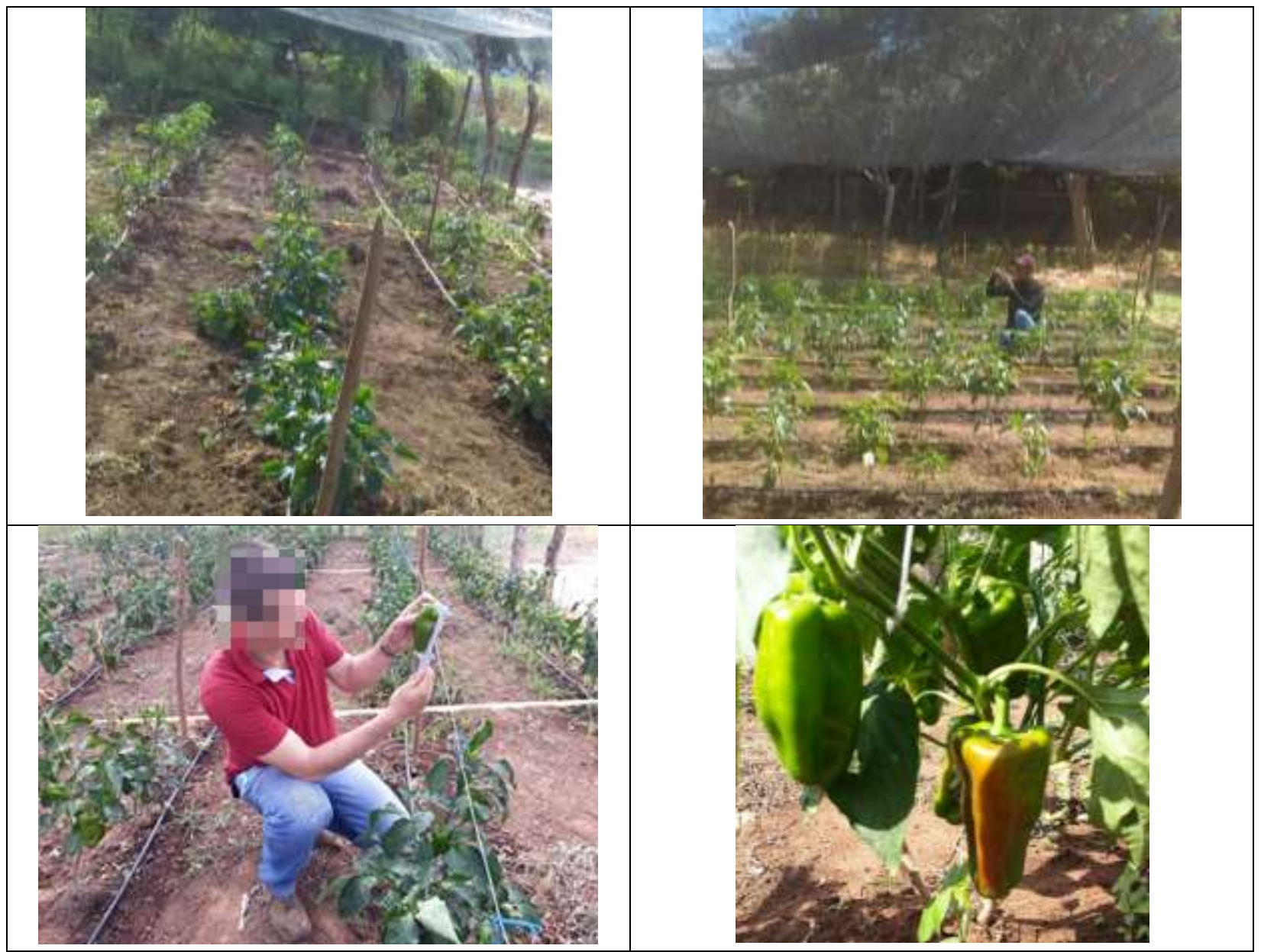

Figura 1. Desarrollo y seguimiento del cultivo de pimentón. Fuente: Autor, Salazar (2020).
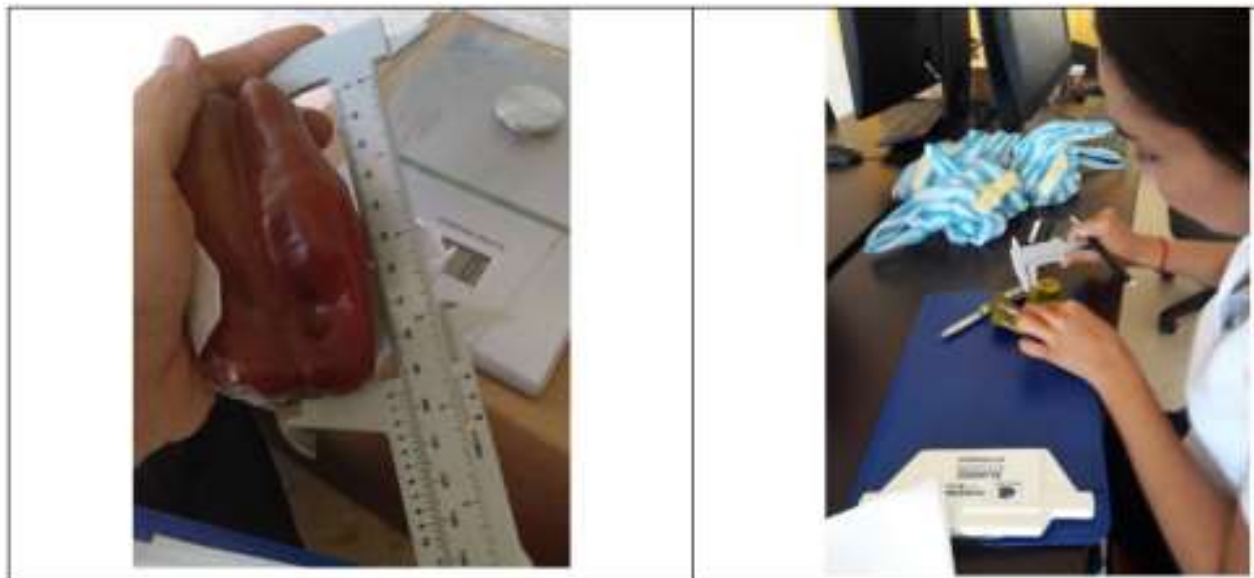

Figura 2. Medición de las variables: diámetro polar y grosor de la pulpa. Fuente: Autor, Salazar (2020). 


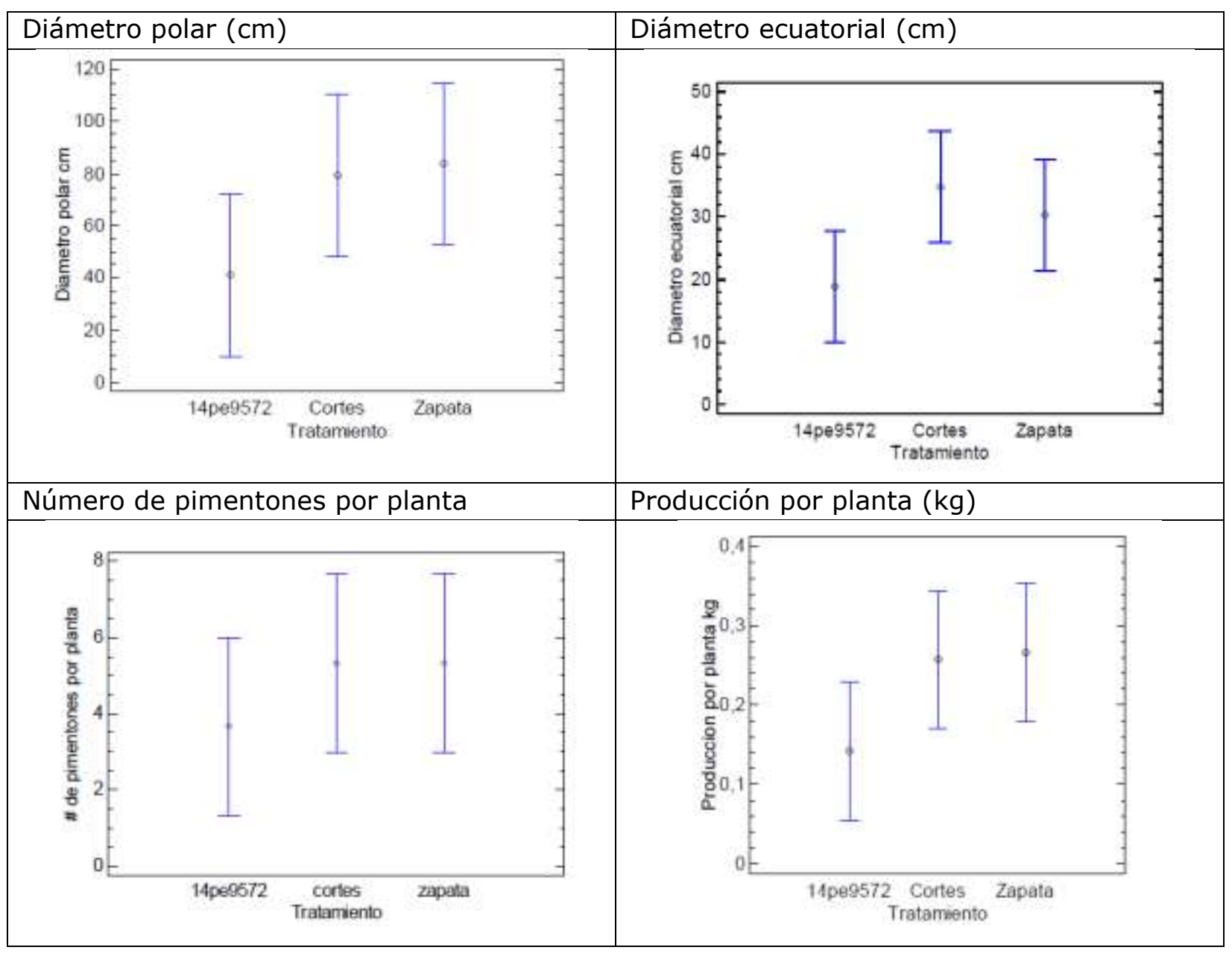

Figura 3. Análisis comparativo de las variables. Fuente: autores.

\section{CONCLUSIONES}

Con base en los resultados de esta investigación sobre las características agronómicas del cultivo de ají pimentón (Capsicum annuum) en el municipio de Valledupar - Cesar, se puede concluir que la evaluación de los parámetros establecidos en esta investigación tales como: diámetro polar, diámetro ecuatorial, producción de pimentones por planta, número de pimentones por planta y grosor de la pulpa de las variedades Cortes, Zapata, y 14 pe9572 no presentan una diferencia significativa entre las medidas analizadas de cada uno de los tratamientos. Se presenta su valor medio, junto con el $95 \%$ intervalo de confianza.

Se obtuvo una producción de 4,04 t ha- 
1 , que es baja frente al rendimiento nacional, que es de $11 \mathrm{t} \mathrm{ha}^{-1}$, pero según estudios de (Pérez $A$. et al 2018) en la amazonia ecuatoriana, alcanzaron una producción $6,28 \mathrm{t} \mathrm{ha}^{-1}$ a campo abierto, con un número de 6,7 frutos por planta y un peso de fruto de $0,8 \mathrm{~kg}$ por planta, en este sentido las variedades Cortes y Zapata presentaron valores similares con respecto al número de frutos ( 6,8 frutos por planta) pero con un peso menor $(0,35 \mathrm{~kg}$ por planta) que indican que se deben hacer estudios sobre la fertilización y requerimiento hídrico del cultivo para mejorar sus características agronómicas. También se encontró que en Costa Rica (Cabalceta y Monge Pérez. 2017) produjeron bajo invernadero con el genotipo Estrella (variedad de forma rectangular y de casca roja como las tres variedades utilizadas en este estudio), que el pimentón alcanzó 3,38 frutos por planta inferior a los producidos en Valledupar a campo abierto, pero con un peso de $0,8 \mathrm{~kg}$ por planta, que también indica que se tiene una buena producción con respecto al número de frutos por planta, pero que hay que mejorar el peso de los frutos.

\section{REFERENCIAS}

Alemán Pérez, Reinaldo Demesio, Domínguez Brito, Javier, Rodríguez Guerra, Yoel, Soria Re, Sandra, Torres
Gutiérrez, Roldán, Vargas Burgos, Julio César, Bravo Medina, Carlos, \& Alba Rojas, Jorge Luis. (2018). Indicadores morfofisiológicos y productivos del pimiento sembrado en invernadero y a campo abierto en las condiciones de la Amazonía ecuatoriana. Centro Agrícola, 45(1), 14-23. Recuperado en 08 de febrero de 2021, de http://scielo.sld.cu/scielo.php?script=sc i_arttext\&pid=S0253-

$57852018000100002 \& \operatorname{lng}=e s \& t \operatorname{lng}=e s$

ASOHOFRUCOL, 2006. Desarrollo de la fruticultura del Cesar.

http://www.asohofrucol.com.co/archivo s/biblioteca/biblioteca_103_Plan\%20Nal \%20frur-CESAR.pdf

Elizondo-Cabalceta, E; Monge-Pérez, J. Evaluación de rendimiento y calidad de 15 genotipos de pimiento (Capsicum annuum L.) cultivados bajo invernadero en Costa Rica. Recuperado de https://www.scielo.sa.cr/pdf/tem/v30n 4/0379-3982-tem-30-04-3.pdf Tecnología en Marcha. Vol. 30-4. Octubre-Diciembre 2017. Pág 3-14

DANE. 2015. El cultivo de pimentón (Capsicum annuum $\mathrm{L}$ ) bajo invernadero Recuperado de

https://www.dane.gov.co/files/investiga ciones/agropecuario/sipsa/Bol_Insumos jul_2015.pdf

Hueso, A; Cascant, M. 2012. Metodologías y técnicas cuantitativas de investigación. Editorial Universidad Politécnica de Valencia. Recuperado de https://riunet.upv.es/bitstream/handle/ 10251/17004/Metodolog\%C3\%ADa\%20 y\%20t\%C3\%A9cnicas\%20cuantitativas 
\%20de\%20investigaci\%C3\%B3n_6060. pdf?sequence

Martínez A. (2015). Requerimientos nutricionales del ají Capsicum annunm

L. y su relación con rendimiento bajo condiciones ambientales. Recuperado de

http://bdigital.unal.edu.co/48562/1/11 16233280.pdf

Montes A. (2017). El ají dulce (Capsicum annuum) como alternativa de producción agrícola sostenible en el corregimiento de Caracol -Toluviejo, departamento de Sucre. 69.
Recuperado de

http://repository.lasalle.edu.co/bitstrea m/handle/10185/21339/46132060_201 7. pdf? sequence $=1$ \&isAllowed $=y$

Salisburry, F. Ross, C. 2000. Fisiología de las plantas. Recuperado de https://biblioteca.unirioja.es/biba/mas_ info. php?-titn=192711

Valledupar A. d. (2019). Mi municipio. Valledupar. Alcaldía municipal de Valledupar. Recuperado de http://www.valleduparcesar.gov.co/Paginas/default.aspx 